\title{
openheart Sudden Unexpected Death in North Carolina (SUDDEN): methodology review and screening results
}

\author{
Parin P Nanavati, ${ }^{1}$ John Paul Mounsey, ${ }^{1}$ Irion W Pursell, ${ }^{1}$ Ross J Simpson Jr, ${ }^{1}$ \\ Mary Elizabeth Lewis, ${ }^{1}$ Neil D Mehta, ${ }^{1}$ Jefferson G Williams, ${ }^{2,3}$ \\ Michael W Bachman, ${ }^{2,3} \mathrm{~J} \mathrm{Brent} \mathrm{Myers,}^{2,3}$ Eugene H Chung ${ }^{1}$
}

To cite: Nanavati PP Mounsey JP, Pursell IW, et al. Sudden Unexpected Death in North Carolina (SUDDEN): methodology review and screening results. Open Heart 2014;1:e000150. doi:10.1136/openhrt-2014000150

Received 23 May 2014 Revised 31 July 2014 Accepted 4 August 2014

\section{CrossMark}

\footnotetext{
${ }^{1}$ Divisions of Cardiology and Cardiac Electrophysiology, Department of Medicine, UNC Heart and Vascular, University of North Carolina at Chapel Hill, Chapel Hill, North Carolina, USA

${ }^{2}$ Department of Emergency Medical Services, Wake County, Raleigh, North Carolina, USA

${ }^{3}$ UNC Department of Emergency Medicine, UNC$\mathrm{CH}$, Chapel Hill, North Carolina, USA
}

Correspondence to Dr Eugene $\mathrm{H}$ Chung; ehchung@med.unc.edu

\section{ABSTRACT}

Objectives: This paper describes the methodology for a prospective, community-based study of sudden unexpected death in Wake County, North Carolina. Methods: From 1 March to 29 June 2013, data of presumed cardiac arrest cases were captured from Wake County Emergency Medical Services. Participants were screened into the presumed sudden unexpected death group based on specific and sequential screening criteria, and medical and public records were collected for each participant in this group. A committee of independent cardiologists reviewed all data to determine final inclusion/exclusion of each participant into registry.

Results: We received 398 presumed cardiac arrest referrals. Of these, 105 participants, age 18-65 years old, were identified as presumed sudden unexpected deaths. The primary reason for exclusion was survival to hospital $(38 \%)$. Ninety-five per cent of participants in the presumed sudden unexpected death group experienced an unwitnessed death. Hypertension was present in almost $50 \%$, while dyslipidaemia and diabetes mellitus were present in almost $25 \%$ of the same group. In addition, the presumed sudden unexpected death group includes $67.6 \%$ males $(95 \%$ $\mathrm{Cl} 58$ to 76 ) whereas the control group only included $58.9 \%$ (95\% Cl 46 to 55 ) males.

Conclusions: Participant identification and data collection processes identify presumed sudden unexpected death cases and secure medical and public data for screening and final adjudication. The study infrastructure developed in Wake County will allow its expansion to other counties in North Carolina. Preliminary data indicate the study presently focuses on a population demographically representative of North Carolina.

\section{INTRODUCTION}

Sudden cardiac death, also known as sudden unexpected death (SUD) is the leading cause of death worldwide but the precise incidence remains undefined. ${ }^{1}$ For example, in the USA, a study using national data and the WHO definition of sudden death

\section{KEY MESSAGES}

What is already known about this subject?

- Sudden cardiac death, also known as sudden unexpected death, is the most common cause of death in the western world. Current estimates of the annual incidence of sudden cardiac death vary widely due to inconsistent definitions and non-uniform means of case ascertainment. A positive family history for sudden unexpected death, depressed left ventricular function, coronary heart disease, hypertension, diabetes mellitus and inherited channelopathies are some of the most important risk factors for sudden unexpected death.

What does this study add?

- Previous cohort studies of sudden death victims have been performed on middle-class and largely Caucasian populations, leading to likely under-reporting of cases among socioeconomically diverse populations and minorities. The Sudden Unexpected Death in North Carolina (SUDDEN) study aims to increase our understanding of the causes of sudden unexpected death and identify high-risk populations. By utilising a prospective, community-wide methodology, we aim to discover meaningful predictors of sudden unexpected death and determine incidence in the diverse population of North Carolina in the USA.

How might this impact on clinical practice?

- By increasing our understanding of the epidemiology and potential causes of sudden unexpected death, clinicians and policymakers will be able to develop individual, community and regional interventions to reduce sudden unexpected death incidence. Risk stratification strategies based on multiple variables will enable early identification and intervention in diverse populations.

estimated an incidence of $456076 .^{2}{ }^{3}$ Conversely, a population-based study from Oregon projected a much lower national 
incidence at $180000-250000 .{ }^{4}$ The true international incidence of SUD is unknown and our understanding of its effect on national health is limited, restricting ability to identify groups that are most vulnerable based on socioeconomic status and health history.

The variability in estimates is related to the definition of SUD utilised, the source of the data and the population studied. ${ }^{5}$ The WHO defines sudden death as a witnessed sudden, unexpected death within $1 \mathrm{~h}$ of symptom onset or, if unwitnessed, within $24 \mathrm{~h}$ of the victim having been observed alive and symptom free. ${ }^{6}$ This is used as a working definition in many studies, but inevitably its use will exclude many potential cases. The terms sudden cardiac arrest, sudden cardiac death, SUD and broadly, sudden death may have different definitions and are often used interchangeably. The overlapping nature of definitions and interchangeability of the terminology associated with sudden death may in part have led to the wide variability in reported incidence rates. Data sources used also vary. Death certificate data are often used to calculate incidence rates in place of prospective population surveillance but the accuracy of death certificate data is limited. ${ }^{7-9}$ Finally, the estimate of incidence will depend on the population studied. Previous cohort studies of sudden death including the SUDS study from Multnomah County, Oregon, USA, and The Maastricht Circulatory Arrest Registry study from the Netherlands have included largely Caucasian populations potentially leading to underreporting of cases among socioeconomically deprived populations and minorities, particularly those of African and Afro-Caribbean descent. ${ }^{111}$

Previous research of risk factors for sudden death may also have suffered from these variable definitions and non-representative sampling. Identified comorbidities for sudden death include coronary heart disease, hypertension, diabetes mellitus, dyslipidaemia and ventricular hypertrophy. ${ }^{12}{ }^{13}$ A family history of sudden death has been shown to predispose individuals to experiencing sudden death, but there are only a few genomic studies of sudden death. ${ }^{14} 15$ The use of postmortem ultrasounds to detect heart abnormalities, such as pericardial effusion, myocardial rupture and ventricular hypertrophy, has been studied but not implemented in this population. ${ }^{16}$ Assessing these potential risk factors within a representative ethnically and socioeconomically diverse sample of sudden death cases is critical to understanding true physiological causes of SUD and developing strategies for prevention.

The overall purpose of the Sudden Unexpected Death in North Carolina (SUDDEN) study is to address these concerns by elucidating the epidemiological, genetic and pathophysiological causes of SUD in North Carolina (NC). The NC population encompasses areas along the eastern coast with increased rates of cardiovascular disease and stroke (within the 'Stroke Belt'), ${ }^{17}$ and a western region with stroke rates similar to the national average. ${ }^{18}$ The pilot county, Wake, contains the state's capital city of Raleigh and includes a demographically diverse population that is representative of the national population. ${ }^{19}$ We aim to clarify risk factors, estimate incidence and estimate underlying pathophysiology of SUD by sampling broadly in a diverse, representative population. The purposes of the present study are to describe the methodology used to ascertain, screen and adjudicate SUD cases in Wake County, NC, and to describe this preliminary presumed SUD cohort.

\section{METHODS \\ Case ascertainment}

Referrals are obtained from Emergency Medical Services (EMS). We began the study in Wake County (2012 population: 952 151). ${ }^{19}$ Potential cases of SUD are initially identified by electronic query of the Wake County EMS patient care reporting software (ESO Solutions V.4.8, Austin, Texas, USA). Charts are identified as possible cases of SUD if the chart contains a provider primary impression or secondary impression cardiac arrest or obvious death or traumatic circulatory arrest, or if treatment with automated external defibrillator, cardiopulmonary resuscitation (CPR) or manual defibrillation is provided. These broad criteria were chosen to maximise sensitivity for detecting all possible cardiac arrest (and therefore SUD) cases. Twenty-nine relevant data fields from each patient chart meeting the above criteria are then exported into an Excel (Microsoft V.14, Redmond, Washington, USA) spreadsheet and transmitted securely to the study coordinator on a daily basis. All potential enrolees are cross matched electronically with the $\mathrm{NC}$ State Death Certificate Database to ensure accuracy of demographic and death data. The data collection process for each participant file is displayed in figure 1 .

Participants are excluded if any of the following criteria are met: (1) the participant is a survivor: e.g. patient was treated and survived, or transported and survived to emergency department of hospital; (2) the participant was over age 65 years; (3) the participant experienced an expected death: e.g. participant was under hospice care or has valid 'do not resuscitate' order; (4) the participant was not free living: e.g. participant's primary residence was a skilled nursing facility; (5) the participant died due to an obvious non-cardiac cause of death: e.g. trauma, violent death, overdose, drowning or suicide; (6) the participant was under age 18 years; (7) the participant was not a resident of NC. Survivors are considered a distinct group of SUD victims, which makes up a small proportion of SUDs. ${ }^{20}$ These patients are tracked but not reported in this paper. Screening is completed independently by two trained research assistants: their data entry is verified and the study coordinator resolves any discrepancies.

\section{Medical record request}

Death certificate data are procured electronically from the NC Center for Health Statistics to identify the death certificate signer ('death attendant'). A medical record 
Figure 1 Data procurement timeline. This figure shows the process of case ascertainment and data collection.

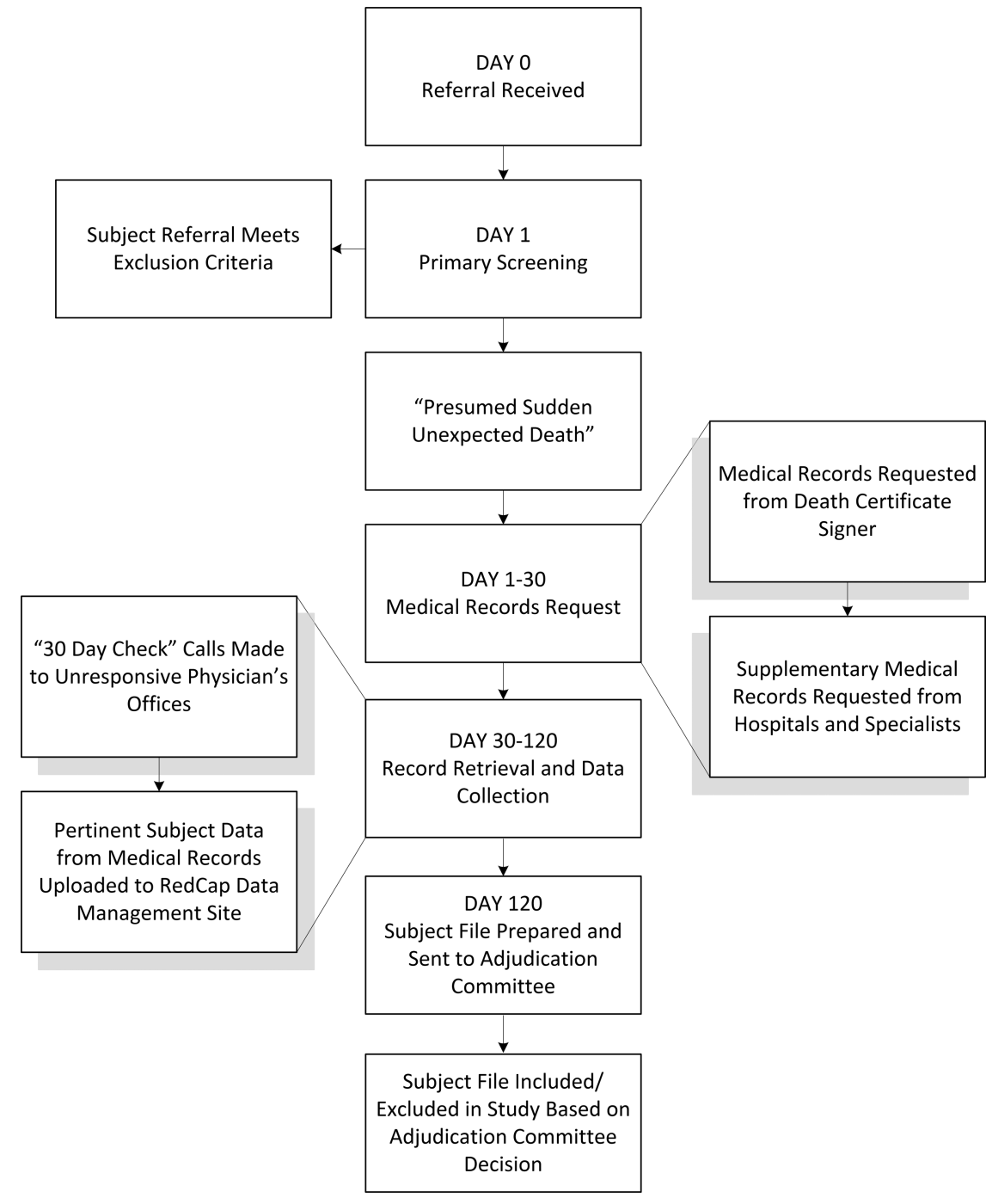

request is sent to this death attendant. For participants without a physician death attendant, we request all reports from the NC Medical Examiner's Office. Additionally, medical record requests are sent to local hospitals for newly screened participants each week. Received records are systematically reviewed to determine whether the participant was treated by other providers and requests are sent to these providers.

If no medical records are received 30 days after an initial request, a follow-up call is placed to the physician's office. Additional public information is collected for the participant, including death notices and other news reports. All collected information is assembled into a relational database.

\section{Adjudication}

An Adjudication Committee, comprising of 10 independent cardiologists, reviews all cases screened into the study. Three members are randomly assigned to conduct an independent review of each file to determine (1) whether the patient's terminal event is SUD and (2) the cause (s) of death.
Participant files of all collected data are distributed to the Adjudication Committee members for final review when the following criteria are met: (1) participant was not excluded during screening, (2) death certificate for the participant is on file, (3) death attendant records have been received or it is determined that no additional data are available. In the event of a disagreement regarding inclusion/exclusion of a specific case, the determination is based on the majority opinion. Inter-rater variability and agreement are calculated for each case and all members of the Adjudication Committee individually.

\section{Data management}

Study data are collected and entered into REDCap (Research Electronic Data Capture). The study data manager and two data entry technicians are responsible for the data entry process. All data are entered using Single Key Entry Protocol (error rate 0.370). ${ }^{21}$ When the data manager completes validation process, the record status is changed from 'Unverified' to 'Complete' and is locked to prevent any changes. 


\section{Statistical methods}

This paper reports study data acquired from 1 March to 29 June 2013. Demographic data from death certificates were collected for all Wake County deaths between the ages of 18 and 65 years during the same time period and CIs were calculated for these data points for comparison.

\section{Genomics}

We will collect genetic material from select participants with medical examiner signed death certificates. DNA will be extracted, prepared for whole exome sequencing and processed at the UNC High Throughput Sequencing Center. Output files will be processed through Renaissance Computing Institute at UNC in order to identify variants of likely clinical significance and linked with clinical data collected in REDCap. Families are offered the option to be notified of any pertinent findings and are then able to contact the study geneticist for a consultation.

\section{Postmortem ultrasound}

We will perform cardiac ultrasounds on select medical examiner cases in order to collect data on the prevalence of major cardiac structural abnormalities in victims of SUD. Study technicians will collect ultrasound images using standard two-dimensional echocardiograph technology. Images will be reviewed by a study investigator who is also a board-certified cardiologist. We chose to limit these examinations to medical examiner cases because such cases provide a cohort for which data can be acquired consistently.

\section{Governance}

The protocols for this study have been exempted by the UNC Institutional Review Board and reviewed by the UNC Hospital Bio-Ethics Committee. The Steering Committee, composed of four cardiologists and a registered nurse, is charged with study management and oversight. An Internal Ethics Committee reviews any issues that may affect the ethical acceptability of the research in the community. The Writing Committee oversees all aspects of data access, study publications and presentations. The Quality Assurance Committee audits the study processes to ensure data quality and verifiable data collection.

\section{RESULTS}

\section{Initial screening process}

From 1 March to 29 June 2013, we received 398 referrals from Wake County EMS. Of these referrals, 293 (74\%) were excluded during primary screening. Of the 293 excluded, $150(51 \%)$ were excluded because they survived to the hospital emergency department and 122 referrals $(42 \%)$ were excluded because they were over age 65 at the time of death. Of the remaining 21 excluded, 5 deaths were expected and 8 were due to non-cardiac aetiology. The final 8 excluded were minors or non-residents of NC. Figure 2 details the number of referrals meeting each exclusion criterion.

\section{The presumed sudden unexpected death cohort}

The screening process identified 105 of 398 referrals as presumed SUD participants. Demographic data were collected from the NC Department of Health Statistics for these participants. Demographic data were collected for all Wake County deaths between the ages of 18 and 65 years, the control group, during the same time frame. The control group had an average age of death of 52.5 years (95\% CI 51.4 to 53.5) and the presumed SUD group had an average age of death of 49 years (95\% CI 47.5 to 52.2$)$. There was a higher percentage of males in the presumed SUD group than in the control group, $67.6 \%$ (95\% CI 58\% to $76 \%$ ) and $58.9 \%$ (95\% CI $46 \%$ to $55 \%$ ), respectively.

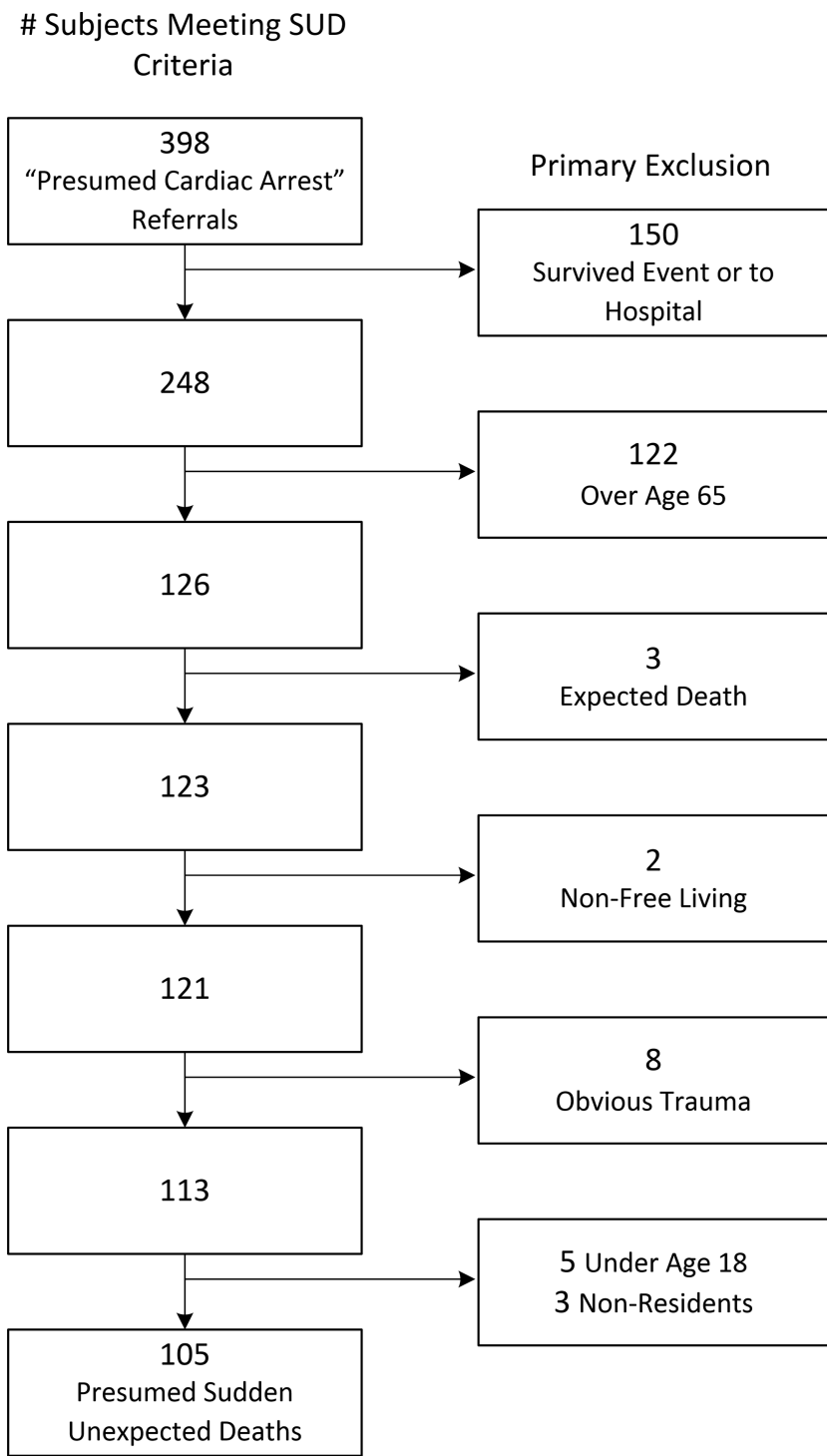

Figure 2 Exclusion criteria and number of referrals meeting primary exclusion. This figure shows the order of the sequential exclusion criteria and the number of referrals meeting each criterion for referrals received from 1 March to 29 June 2013 (SUD, sudden unexpected death). 
Figure 3 Demographic data for presumed sudden unexpected death group, Wake County deaths ages 18-65 years and total Wake County deaths. This figure shows data collected from State Center for Health Statistics for deaths from 1 March to 29 June 2013.

\section{Demographics of Death Groups}

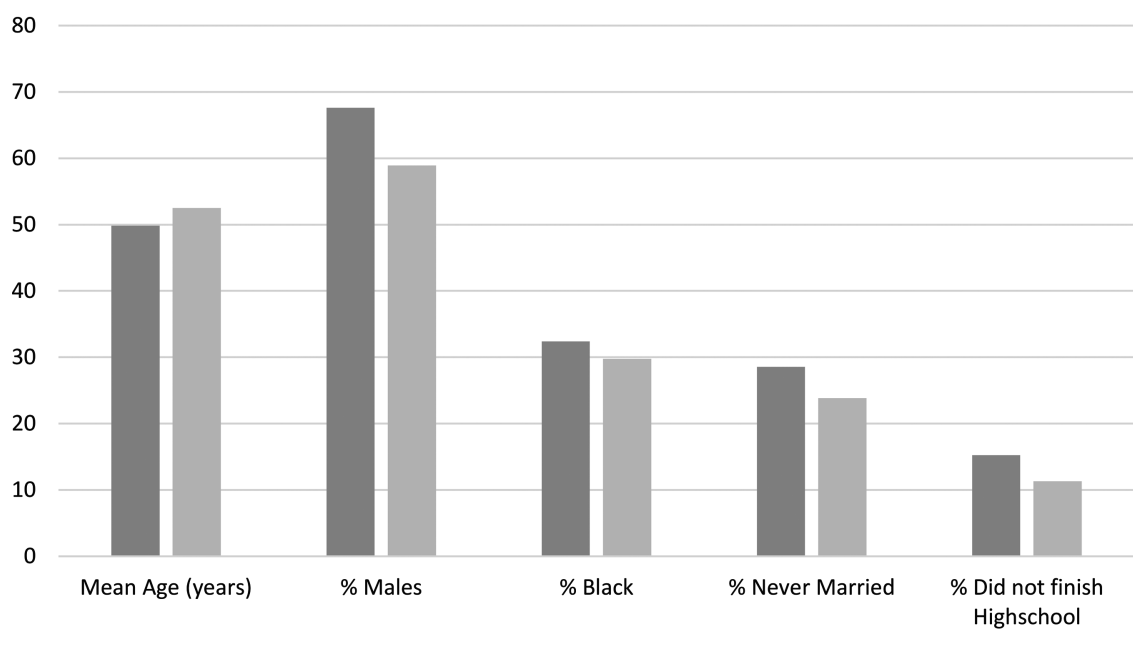

Presumed Sudden Death Group Wake County Deaths Ages 18-65
The per cent of African-Americans, single participants and participants with less than a high-school education is similar in both these groups (figure 3).

A systematic review of medical records was completed to determine the prevalence of comorbidities associated with SUD. The results are displayed in table 1. National prevalence is displayed for comparison. Forty-nine participants $(52 \%)$ had history of hypertension, $22(23 \%)$ had diabetes mellitus, $23(24 \%)$ had history of dyslipidaemia, $18(19 \%)$ had coronary heart disease and 20 $(21 \%)$ had a noted cardiomyopathy.

Table 1 Comorbidities of presumed sudden unexpected death participants and national incidence rates

\begin{tabular}{llc}
\hline & Per cent & $\begin{array}{l}\text { National } \\
\text { prevalence (\%)* }\end{array}$ \\
\hline Hypertension & $56.8 \dagger$ & $28.6 \ddagger$ \\
Diabetes mellitus & $27.4 \S$ & $6.4 \rrbracket$ \\
Dyslipidaemia & $30.5^{\star \star}$ & $13.8 \dagger \dagger$ \\
Coronary heart disease & $22.1 \neq \ddagger$ & $6.4 \S \S$ \\
Cardiomyopathy & $24.2 \eta\rceil$ & $0.2^{\star \star *}$ \\
\hline
\end{tabular}

Many participant records indicated more than one comorbidity, and all are listed. The percentages were taken of the 95 participants with medical data.

*Data in this column are from source 22.

†Participants with a clinical history of hypertension.

$\ddagger$ Prevalence among adults over 18 years in 2010 .

IPrevalence among adults $>20$ with physician-diagnosed diabetes mellitus in 2010.

§Participants with medical records with noted use of antidiabetic medications or clinical history of diabetes mellitus.

${ }^{\star *}$ Participants with defined clinical history or use of lipid-lowering medications.

$\dagger+$ Hypercholesteraemia prevalence among adults $>20$.

$\ddagger \ddagger$ Participants with physician noted history of coronary artery bypass grafting, myocardial infarction, pathological $Q$ waves on

ECG, history of myocardial infarction, angina or positive stress test results.

$\S \S$ Coronary heart disease prevalence among adults $>20$.

Iी|Participants with medical records in which physician report of

ischaemic, dilated, hypertrophic or restrictive cardiomyopathy.

***Hypertrophic cardiomyopathy prevalence in US population.
Of the 105 presumed SUD participants, 88 (84\%) experienced the terminal event at their primary residence and $15(14 \%)$ in a public area. Death was unwitnessed for 100 participants (95\%). Of these 100 deaths, it was unknown when 84 participants were last seen alive, 11 were last seen alive within a day and 5 were last seen alive over $24 \mathrm{~h}$ before death. Table 2 describes location and witness status of these deaths.

Death certificate data from the NC Center for Health Statistics were procured for all SUD participants: based on International Classification of Diseases V.10 (ICD-10) codes from the death certificates, $73(70 \%)$ experienced a natural cause of death and $32(30 \%)$ died from external or unknown reasons. Sixty-six participants (63\%) had medical examiner signed death certificates and the remaining 39 participants $(37 \%)$ had physician signers.

Table 2 Emergency Medical Service (EMS) referrals for presumed sudden unexpected death participants referred from 1 March to 29 June 2013

\begin{tabular}{lrl}
\hline & N & Per cent \\
\hline Death certificate available & 105 & $(100)$ \\
Death at primary residence & 88 & $(83.8)$ \\
Death in public area* & 15 & $(14.3)$ \\
Death unwitnessed & 100 & $(95.2)$ \\
Unknown hours since last seen alive $\dagger$ & 84 & $(84.0)$ \\
Last seen alive less than 24 h before & 11 & $(11.0)$ \\
death† & & \\
Last seen alive over 24 h before death $\dagger$ & 5 & $(5.0)$ \\
Medical data collected & 95 & $(90.5)$ \\
ECG collected & 26 & $(24.8)$ \\
\hline
\end{tabular}

EMS's chief narratives were examined for witnessed/unwitnessed data; if narrative did not indicate that the death was witnessed, the death was considered unwitnessed.

*Location was considered public area if it was not the participant's primary residence or a healthcare facility, such as a nursing home or assisted living facility.

†These data points were taken for participants with unwitnessed deaths only. 
A full autopsy was performed for a total of 25 participants. Medical records were collected for 95 (91\%) of the participants in the presumed SUD group. Of these, $26(25 \%)$ also had one or more ECG. Table 2 displays these data.

\section{DISCUSSION}

The SUDDEN study identifies and screens presumed SUDs in Wake County, NC. Medical records and death certificate data were collected in a systematic manner that enables us to accurately estimate incidence and to understand the epidemiological, genetic and pathophysiological causes of SUD in a racially and socioeconomically diverse population (figure 1).

Sudden death definitions used in studies are inconsistent, leading to variable incidence rates. In 1985, the WHO Scientific Group on sudden cardiac death advocated to define specific characteristics surrounding cardiac arrest, rather than propose a universal definition. ${ }^{5}$ We chose to study 'SUD', which is a broader term that includes sudden cardiac deaths and other deaths that are sudden and unexpected. We utilised the WHO recommendation to design our methodology to capture and screen SUD participants in NC.

This medical record-based methodology is likely to lead to differing incidence rates than previous studies. An epidemiological study of sudden death states that limiting the definition of sudden death to those that occur within the WHO definition time restrictions might be too restrictive. ${ }^{2}$ The SUDDEN study employs a medical record-driven case inclusion process that we believe will produce a more comprehensive registry of SUD cases than previous studies (figure 2). Of the 100 participants who experienced unwitnessed deaths, it was unknown when 84 were last seen alive (table 2). The Oregon study would exclude these 84 participants based on the WHO definition. ${ }^{23}$ We exclude participants over 65 years old and those in nursing facilities, presuming these groups have an increased likelihood of experiencing an expected death, to focus our study on a younger cohort that are likely victims of an unexpected event. ${ }^{24}$ This age limit accounted for $42 \%$ of our exclusions (figure 2). Additionally, we are inclusive of cases of trauma in which the trauma may have been the result of a cardiac event, as shown by high percentage of external or unknown causes of death $(30 \%)$. The screening criteria outlined above will allow us to describe SUD in a clinically meaningful group.

Overall, the Wake County and NC populations are more racially diverse and more demographically representative of the US population than the Multnomah County population studied in the Oregon study (table 3). We have identified a young, racially diverse population that is experiencing SUD; preliminary data show that there is a high per cent of males and participants with history of common comorbidities who are experiencing presumed SUD in this population (table 1).
Table 3 US Census Data for North Carolina, Wake County, North Carolina and Multnomah County, Oregon

\begin{tabular}{|c|c|c|c|}
\hline Location & $\begin{array}{l}\text { Population } \\
(2010)\end{array}$ & $\begin{array}{l}\text { Per cent } \\
\text { white } \\
(\mathbf{2 0 1 2})\end{array}$ & $\begin{array}{l}\text { Per cent } \\
\text { black } \\
(2012)\end{array}$ \\
\hline USA & 308745538 & 77.9 & 13.1 \\
\hline North Carolina & 9535483 & 71.9 & 22.0 \\
\hline $\begin{array}{l}\text { Wake County, North } \\
\text { Carolina }\end{array}$ & 900993 & 69.6 & 21.4 \\
\hline $\begin{array}{l}\text { Multnomah County, } \\
\text { Oregon* }\end{array}$ & 660486 & 79.2 & 5.7 \\
\hline
\end{tabular}

Our presumed SUD group was compared with residents in Wake County ages 18-65 years who died during the same time frame. The control group and the presumed SUD group are demographically similar, except for gender (figure 3). These preliminary data are similar to findings of the Oregon study, which found a greater proportion of males than females in the $<65$ years age range. ${ }^{23}$ There is a minimal difference between the age at death of the control group and the presumed SUD group, 52.5 and 49.8 years, respectively.

Previous studies have determined common conditions related to sudden death. Among the presumed SUD group, there is a high proportion of hypertension, dyslipidaemia and coronary heart disease when compared with the national prevalence data (table 1). As expected based on previous reports, there is a greater percentage of the presumed SUD group with all of these comorbidities than reported in the national population. ${ }^{12} 2223$ Collecting demographic data and medical histories will enable us to develop predictive models and risk stratification tools for SUD. ${ }^{25}$

\section{Limitations}

There are limitations to our methodology. First, we may not have captured all SUD cases in Wake County. While the large majority of out-of-hospital deaths that would qualify as SUDs in Wake County are evaluated by EMS, there remains a possibility that some deaths would be missing from EMS records. In addition, in some cases of obvious death that might qualify as SUD, EMS documentation is limited due to brief EMS involvement (e.g. a potential crime scene). We believe this to be a small number compared with the overall population and the sample obtained is likely to be representative of the $\mathrm{NC}$ population.

Second, not all potential SUD events are included in our out-of-hospital SUD cohort as we excluded survivors, individuals who survived to but then died in hospital, institutionalised participants, non-residents, minors and individuals over 65 years old. By eliminating these groups, we may introduce selection bias. We keep records of all participants referred to the study and the noted reasons for exclusion. Once our data collection 
methodology is well established, including these groups and considering them in an analysis of possible SUD may be a topic for future study.

Despite our efforts, medical records are not available for all presumed SUD participants. Additionally, limited medical information is available for participants who did not make recent visits to a healthcare provider. However, our protocol aims to build as comprehensive a medical database as possible for SUD victims in NC.

\section{CONCLUSION}

We have developed a sound epidemiological approach to collect data on SUD that we have piloted in one large county and which is generalisable to other counties in NC. The preliminary data indicate that we have a population demographically representative of $\mathrm{NC}$ and the overall national population with similar trends to previous SUD studies in terms of gender differences and prevalence of comorbidities. We thus believe the SUDDEN study will achieve its overarching missions to improve identification of at risk populations and to guide improved prevention strategies for populations most at risk for SUD.

Collaborators Mark Massey, MD, Epidemiology; Daniel Lackland, MD, Epidemiology; Jim Evans, MD, Genomics; Michael Wagner, PhD, Genomics.

Contributors PPN designed data collection tools, collected and analysed data, prepared and revised the manuscript. JPM designed the study, monitored data collection, revised the manuscript. IWP monitored data collection, implemented the study in Wake County, revised the manuscript. RJS revised the manuscript. MEL collected and analysed data in order to prepare initial tables and figures, revised the manuscript. NDM collected data, revised the manuscript. JGW, MWB and JBM provided data, data interpretation, guidance for study development and revised the manuscript. EHC designed the study, monitored data collection and revised the manuscript.

Funding The SUDDEN study is funded by individual donations from Cecil Sewell, Scott Custer and Joe and Ann Lamb, additional support is provided by The Heart and Vascular Division of the University of North Carolina at Chapel Hill.

\section{Competing interests None.}

Ethics approval The SUDDEN study protocols and methods were reviewed by the UNC Center for Bioethics prior to initiation of data collection. Ongoing ethics review is provided by the SUDDEN Ethics Committee (SEC). The SEC reviews protocols, methods, data output and publications on a semiannual basis or as requested from SUDDEN investigators.

Provenance and peer review Not commissioned; externally peer reviewed.

Data sharing statement No additional data are available.

Open Access This is an Open Access article distributed in accordance with the Creative Commons Attribution Non Commercial (CC BY-NC 4.0) license, which permits others to distribute, remix, adapt, build upon this work noncommercially, and license their derivative works on different terms, provided the original work is properly cited and the use is non-commercial. See: http:// creativecommons.org/licenses/by-nc/4.0/

\section{REFERENCES}

1. Estes NAM. Predicting and preventing sudden cardiac death. Circulation 2011;124:651-6.

2. Adabag AS, Luepker RV, Roger VL, et al. Sudden cardiac death: epidemiology and risk factors. Nat Rev Cardiol 2010;7:216-25.

3. Zheng Z, Croft JB, Giles WH, et al. Sudden cardiac death in the United States, 1989 to 1998 . Circulation 2001;104:2158-63.

4. Kong MH, Fonarow GC, Peterson ED, et al. Systematic review of the incidence of sudden cardiac death in the United States. J Am Coll Cardiol 2011;57:794-801.

5. Deo R, Albert CM. Epidemiology and genetics of sudden cardiac death. Circulation 2012;125:620-37.

6. Zaijia C, James TN, Kulbertus $\mathrm{H}$, et al. Sudden cardiac death. World Health Organ Tech Rep 1985;726:5-25.

7. Myerburg RJ. Scientific gaps in the prediction and prevention of sudden cardiac death. $J$ Cardiovasc Electrophysiol 2002;13:709-23.

8. Every NR, Parsonsc L, Hlatky MA, et al. Use and accuracy of state death certificates for classification of sudden cardiac deaths in high-risk populations. Am Heart J 1997;134:1129-32.

9. Iribarren C, Crow R, Hannan MP, et al. Validation of death certificate diagnosis of out-of-hospital sudden cardiac death. Am J Cardiol 1998;82:50-3.

10. Chugh SS, Reinier K, Teodorescu C, et al. Epidemiology of sudden cardiac death: clinical and research implications. Prog Cardiovasc Dis 2008;51:213-28.

11. de Vreede-Swagemakers JJM, Gorgels APM, Dubois-Arbouw WI, et al. Out-of-hospital cardiac arrest in the 1990s: a population-based study in the Maastricht area on incidence, characteristics and survival. J Am Coll Cardiol 1997;30:1500-5.

12. Jouven X, Desnos M, Guerot C. Predicting sudden death in the population: the Paris Prospective Study I. Circulation 1999;99:1978-83.

13. Chugh SS, Kelly KL, Titus JL. Sudden cardiac death with apparently normal heart. Circulation 2000;102:649-54.

14. Friedlander $Y$, Siscovick DS, Weinmann $S$, et al. Family history as a risk factor for primary cardiac arrest. Circulation 1998;97:155-60.

15. Darbar D. Genomics, heart failure and sudden cardiac death. Heart Fail Rev 2010;15:229-38.

16. Uchigasaki S, Oesterhelweg L, Gehl A, et al. Application of compact ultrasound imaging device to postmortem diagnosis. Forensic Sci Int 2004;140:33-41.

17. Alberts MJ. The stroke belt consortium. $J$ Stroke Cerebrovasc Dis 1996;6:54-8.

18. Howard G, Evans GW, Pearce K, et al. Is the stroke belt disappearing? An analysis of racial, temporal, and age effects. Stroke 1995;26:1153-8.

19. U.S. Census Bureau State \& County Quickfacts. http://quickfacts. census.gov (accessed Apr 2014)

20. Finzi A, Manfredini R, Montanari P, et al. Management of sudden cardiac death survivors. Heartweb 1998;4-8.

21. Paulson A, Overgaard S, Lauritsen JM. Quality of data entry using single entry, double entry and automated forms processing - an example based on a study of patient-reported outcomes. PLOS ONE 2012;7:e35087.

22. Go AS, Go A, Mozaffarian D, et al. Heart disease and stroke statistics-2014 update: a report from the American Heart Association. Circulation 2014;129:28-292.

23. Chugh SS, Jui J, Gunson K, et al. Current burden of sudden cardiac death: multiple source surveillance versus retrospective death certificate-based review in a large U.S. community. J Am Coll Cardiol 2004;44:1268-75.

24. National Center for Health Statistics. Worktable 23R Death rates by 10-year age groups: United States and each state, 2007. http://www. cdc.gov/nchs/data/dvs/MortFinal2007_Worktable23r.pdf (accessed Apr 2014)

25. Fishman Gl, Chugh SS, DiMarco JP, et al. Sudden cardiac death prediction and prevention: report from a National Heart, Lung, and Blood Institute and Heart Rhythm Society Workshop. Circulation 2010;122:2335-48. 